\title{
T1-Weighted Dynamic Contrast-Enhanced MR Perfusion Imaging Characterizes Tumor Response to Radiation Therapy in Chordoma
}

\author{
(D) P. Santos, (D).K. Peck, DJ. Arevalo-Perez, (D) S. Karimi, (DE. Lis, (D)Y. Yamada, (D)A.I. Holodny, and (D). Lyo

\begin{abstract}
BACKGROUND AND PURPOSE: Chordomas notoriously demonstrate a paucity of changes following radiation therapy on conventional MR imaging. We hypothesized that dynamic contrast-enhanced MR perfusion imaging parameters of chordomas would change significantly following radiation therapy.
\end{abstract}

MATERIALS AND METHODS: Eleven patients with pathology-proved chordoma who completed dynamic contrast-enhanced MR perfusion imaging pre- and postradiation therapy were enrolled. Quantitative tumor measurements were obtained by 2 attending neuroradiologists. ROls were used to calculate vascular permeability and plasma volume and generate dynamic contrast-enhancement curves. Quantitative analysis was performed to determine mean and maximum plasma volume and vascular permeability values, while semiquantitative analysis on averaged concentration curves was used to determine the area under the curve. A Mann-Whitney $U$ test at a significance level of $P<.05$ was used to assess differences of the above parameters between pre- and postradiation therapy.

RESULTS: Plasma volume mean (pretreatment mean $=0.82$; posttreatment mean $=0.42$ ), plasma volume maximum (pretreatment mean $=3.56$; posttreatment mean $=2.27$ ), and vascular permeability mean (pretreatment mean $=0.046$; posttreatment mean $=0.028$ ) in the ROIs significantly decreased after radiation therapy $(P<.05)$; this change thereby demonstrated the potential for assessing tumor response. Area under the curve values also demonstrated significant differences $(P<.05)$.

CONCLUSIONS: Plasma volume and vascular permeability decreased after radiation therapy, suggesting that these dynamic contrastenhanced MR perfusion parameters may be useful for monitoring chordoma growth and response to radiation therapy. Additionally, the characteristic dynamic MR signal intensity-time curve of chordoma may provide a radiographic means of distinguishing chordoma from other spinal lesions.

ABBREVIATIONS: $\mathrm{AUC}=$ area under the curve; $\mathrm{DCE}=$ dynamic contrast-enhanced; $K^{\text {trans }}=$ vascular permeability; $\max =$ maximum; $\mathrm{RT}=$ radiation treatment; $\mathrm{V}_{\mathrm{p}}=$ plasma volume

C hordoma is a rare cancer of persistent or ectopic notochord remnants, commonly found in the clivus, spine, and sacrum. ${ }^{1}$ Chordomas account for only $1 \%-4 \%$ of all bone cancers, with an annual incidence of 1 per 1,000,000 and a prevalence of $>1$ per $100,000 .^{2,3}$ Although having a low grade in histology,

Received April 19, 2017; accepted after revision June 15

From the Departments of Radiology (P.S., K.K.P., J.A.-P., S.K., E.L., A.I.H., J.L.), Medical Physics (K.K.P.), and Radiation Oncology (Y.Y.), Memorial Sloan Kettering Cancer Center, New York, New York.

This work was funded, in part, through the National Institutes of Health/National Cancer Institute Cancer Center support grant P30 CA008748.

Please address correspondence to Kyung K. Peck, PhD, Department of Medical Physics and Radiology, Memorial Sloan Kettering Cancer Center, 1275 York Ave, New York, NY 10065; e-mail: peckK@mskcc.org

-- Indicates open access to non-subscribers at www.ajnr.org

三 Indicates article with supplemental on-line tables.

http://dx.doi.org/10.3174/ajnr.A5383 chordomas are locally invasive, aggressive, and prone to recurrence. ${ }^{1}$ Thus, patients are faced with a dismal prognosis and clinical progression mirroring that of malignant neoplasms. The median survival of patients with chordoma is currently 6.29 years, with survival rates dropping from $67.6 \%$ at 5 years to $39.9 \%$ at 10 years. $^{4}$

The diagnosis of chordoma is challenging. Currently, conventional MR imaging is the criterion standard for the primary assessment of chordoma because it allows discrimination of tumor soft tissue from normal architecture, as well as the surveillance of spinal metastases. ${ }^{5-7}$ Classically, chordomas appear isointense or hypointense on T1-weighted MR imaging and hyperintense on T2-weighted MR imaging. ${ }^{8}$ Most lesions exhibit minimum-tomoderate heterogeneous enhancement with gadolinium, mirroring their heterogeneous histology. ${ }^{9}$ Not surprising, conventional MR imaging is limited in its capacity to discriminate chordoma and other spinal cancers. ${ }^{10}$ 
Current treatment guidelines recommend en bloc surgical resection for primary chordoma lesions, followed by adjuvant radiation therapy to eliminate residual disease. ${ }^{1,10}$ Resection quality is largely predictive of postsurgical outcomes: Patients who underwent more aggressive, radical resection exhibited remission lasting nearly 3.5 times longer than those who had only undergone subtotal resection. ${ }^{11}$ Thus, local recurrence despite resection and adjuvant radiation is considered a marker of treatment failure. However, en bloc excision is not universally feasible for all patients with chordoma. Surgical resection of chordoma is often complicated by local invasion of adjacent vessels and impingement of surrounding structures. In these advanced cases, alternative approaches such as subtotal resection, high-dose radiation therapy, and systemic biologic therapies may be indicated. Determining the need for such therapies requires careful monitoring of tumor response to therapy postresection.

However, monitoring tumor progression with conventional MR imaging is complicated by the indolent nature of chordoma: Its lesions are largely clinically silent until later stages of disease, at which point they are resistant to most traditional chemoradiation regimens. ${ }^{1}$ As a result, chordoma lesions typically do not exhibit significant changes in tumor volume in response to therapy, once again limiting the utility of conventional MR imaging in evaluating treatment efficacy and subsequent patient prognosis.

Unlike conventional MR imaging, dynamic contrast-enhanced MR perfusion imaging (DCE-MR imaging) provides insight into the physiologic and hemodynamic characteristics of neoplastic lesions by evaluating tumor vascularity. DCE-MR imaging involves assessing changes in signal intensity with time, before and after contrast injection. ${ }^{12}$ Previous studies have shown that DCE-MR imaging is useful for monitoring the response to therapy in spinal bone metastases, ${ }^{13}$ and for discriminating the hypo- and hypervascular spinal lesions of prostate carcinoma and renal cell carcinoma, respectively. ${ }^{14}$ More recently, DCE-MR imaging has shown clinical utility in chordoma diagnosis, effectively discriminating between chordoma and giant cell tumors of the axial skeleton. ${ }^{15}$

The purpose of this study was to determine whether DCE parameters, plasma volume $\left(\mathrm{V}_{\mathrm{p}}\right)$ and vascular permeability $\left(K^{\text {trans }}\right)$, can be used to monitor tumor response to radiation treatment (RT). We hypothesized that DCE-MR imaging parameters obtained via quantitative and semiquantitative methods can be used to characterize tumor response to RT, detecting subsequent changes in chordoma vasculature, which may later predict parallel changes in tumor volume as detected by conventional MR imaging.

\section{MATERIALS AND METHODS}

\section{Subjects}

We analyzed retrospective radiographic data from patients with pathology-proved chordoma of the sacrum, spine, or clivus. Eleven patients (mean age, 61 years) with DCE-MR perfusion imaging before and after RT were enrolled. Our institutional review board granted a waiver of authorization and consent for this study.

\section{Data Acquisition}

MR imaging sequences were acquired with a $1.5 \mathrm{~T}$ scanner (HDxt 16.0; GE Healthcare, Milwaukee, Wisconsin), equipped with an 8-channel cervical-thoracic-lumbar surface coil. Patients underwent routine MR imaging, including sagittal T1 (FOV, 32-36 cm; section thickness, $3 \mathrm{~mm}$; TR, $400-650 \mathrm{~ms}$; flip angle, $\left.90^{\circ}\right)$ and T2 (FOV, 32-36 cm; section thickness, 3 mm; TR, 3500-4000 ms; flip angle, $90^{\circ}$ ), axial T1 (FOV, $18 \mathrm{~cm}$; section thickness, $8 \mathrm{~mm}$; flip angle, $90^{\circ}$ ) and T2 (FOV, $18 \mathrm{~cm}$; section thickness, $8 \mathrm{~mm}$; TR, $3000-4000 \mathrm{~ms}$; flip angle, $90^{\circ}$ ), and sagittal STIR imaging (FOV, $32-36 \mathrm{~cm}$; section thickness, $3 \mathrm{~mm}$; TR, 3500-6000 ms; flip angle, $\left.90^{\circ}\right)$.

DCE-MR imaging of the spine was acquired on completion of routine MR imaging. A power injector was used to administer a bolus of gadolinium-diethylenetriamine penta-acetic acid at a dose of $0.1 \mathrm{mmol} / \mathrm{kg}$ and rate of $2-3 \mathrm{~mL} / \mathrm{s}$. The kinetic enhancement of tissue before and after contrast injection was obtained by using a 3D T1-weighted fast-spoiled gradient-echo sequence (TR, 4-5 ms; TE, 1-2 ms; section thickness, $5 \mathrm{~mm}$; flip angle, $25^{\circ}$; FOV, $32 \mathrm{~cm}$; matrix, $256 \times 128$; temporal resolution, $6 \sim 7$ seconds) and consisted of 10-12 images in the sagittal plane. Ten phases for preinjection time delay and 30 phases for postinjection were obtained. Sagittal and axial T1-weighted postinjection MR images were additionally acquired after DCE-MR imaging.

\section{Data Analysis}

Data processing and analysis were performed with dynamic image processing software (NordicICE, version 2.3.14; NordicNeuroLab, Bergen, Norway). Preprocessing steps included removal of background noise, spatial and temporal smoothing, and detection of the arterial input function from the aorta. The arterial input function was individually calculated in each acquisition. Pixels with a large change in signal intensity, with a rapid change immediately after bolus injection, and with an early peak in intensity were chosen for the arterial input function. Linear assumption was made between the change in signal intensity and gadolinium concentration to convert the signal intensity curve to a concentration-time curve.

Anatomic images that matched the DCE-MR images were used in ROI placements. Voxel-by-voxel estimates of quantitative perfusion parameters, including vascular permeability and plasma volume, were determined with a Tofts 2-compartment pharmacokinetic model ${ }^{16}$ analysis. The area under the curve (AUC) was obtained from semiquantitative analysis to assess its potential as a discriminator of tumor histology.

ROIs were drawn to encompass the entirety of the chordoma by 2 attending neuroradiologists who were blinded to biopsy results and other clinical data, including age and sex, with careful consideration to avoid lesion margins, normal-appearing marrow, endplates, spondylotic changes, and vessels (including the basivertebral venous plexus). ROI values were determined for each section. Radiologists placing ROIs were blinded to additional clinical information and additional imaging.

\section{Dynamic Concentration-Time Curves}

Dynamic concentration-time curves illustrate changes in MR imaging signal intensity as a function of time. As a semiquantitative 


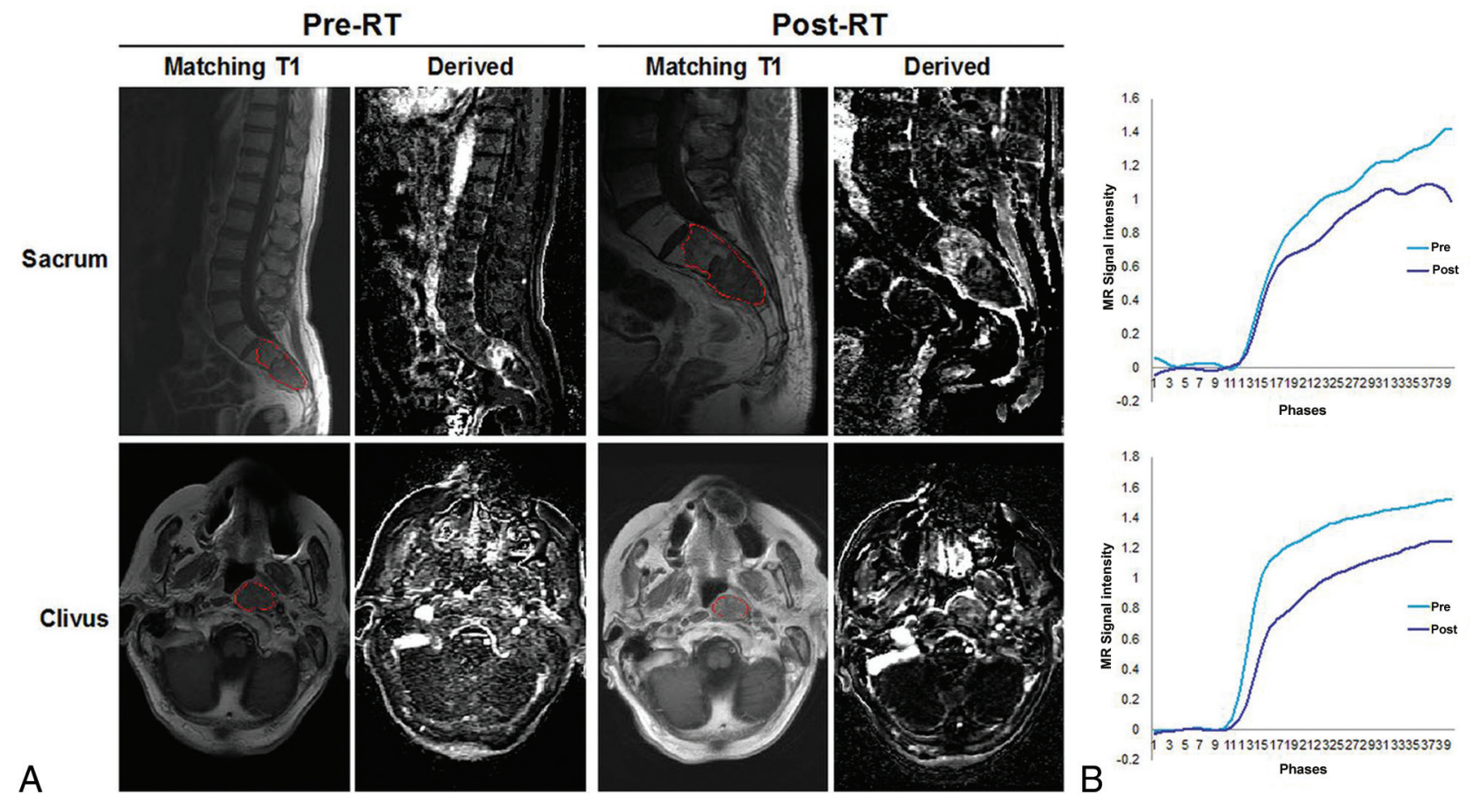

FIG 1. MR signal intensity is diminished in response to radiation therapy in DCE-MR imaging. A, Representative sagittal and axial T1-weighted MR images and dynamic derived images from DCE-MR imaging, illustrating a chordoma of the sacrum and clivus, pre- and postradiation therapy. $B$, Individual dynamic MR signal intensity-time curves pre-RT (blue) and post-RT (purple), obtained from corresponding ROIs (red).

analysis, AUCs in the dynamic concentration-time curves were generated from the same ROIs. Two curves for before and after RT were obtained for each patient. Only MR imaging signal intensities corresponding to the phases of the postinjection period were included for the calculation.

\section{Statistical Analysis}

A Wilcoxon ranked sign test at a significance of $P \leq .05$ was conducted to assess the difference between the $\mathrm{V}_{\mathrm{p}}$ and $K^{\text {trans }}$ maximum $(\max )$ and mean values pre- and post-RT. A MannWhitney $U$ test at a significance of $P \leq .05$ was conducted to assess the difference between the dynamic curves pre- and post-RT.

\section{DCE-MR Imaging Intervals}

There was variability with respect to the number of both pre- and posttreatment DCE-MR imaging perfusion scans provided for each patient. In this study, we selected scans on the basis of the proximity to the time of radiation therapy as measured by pretreatment and posttreatment intervals. The pretreatment interval was measured as the number of days between the most proximal pretreatment DCE-MR imaging study and the first day of radiation therapy; the posttreatment interval was measured as the number of days between the last day of radiation therapy and the most proximal posttreatment DCE-MR imaging study.

\section{Therapeutic Response}

All subsequent posttreatment surveillance MR images and corresponding reports were reviewed. Time to recurrence was measured as the number of days between the last day of radiation therapy to the first radiology report that noted increasing tumor size/signal enhancement concerning for progression. Corresponding radiation oncology notes were reviewed to confirm that the patient's clinical presentation aligned with radiologic findings. For 5 of 11 patients, progression-free survival was measured as the number of days from the last day of radiation therapy until the first surveillance MR imaging that reported possible progression. For the remaining 6 of 11 patients with no concerning radiologic findings, progression-free survival was measured from the last day of radiation therapy until their most recent MR imaging.

\section{RESULTS}

\section{Patient Demographics and Tumor Characteristics}

Six patients had spinal lesions, 4 had sacral lesions, and 1 had a clival lesion. Of the 11 lesions analyzed, 7 were localized and 4 were metastatic. Five lesions were recurrent (On-line Table 1). The average bidimensional size for readers 1 and 2 was $22.9 \times 40.0$ $\mathrm{cm}^{2}$ and $22.7 \times 40.0 \mathrm{~cm}^{2}$ pretreatment, respectively, and $23.6 \times$ $42.2 \mathrm{~cm}^{2}$ and $23.8 \times 40.8 \mathrm{~cm}^{2}$ posttreatment, respectively. The average percentage change post-RT was $4.3 \%$ for reader 1 and $3.4 \%$ for reader 2 . There was good interreader reliability across tumor measurements $\left(R^{2}=1.0\right.$, On-line Table 1$)$.

\section{Vascular Permeability and Plasma Volume}

T1-weighted and corresponding DCE perfusion images are presented in Fig 1A, illustrating 2 representative chordoma cases of the sacrum and clivus, pre- and posttreatment. The averaged dynamic concentration time curves in the ROI are presented in Fig $1 B$.

As expected, chordoma lesions were hypointense or isointense on T1 and hyperintense on T2 (Fig 1A). The overall amplitude of the dynamic concentration time curves is lower at posttreatment. On evaluation of perfusion images, we found that before treat- 


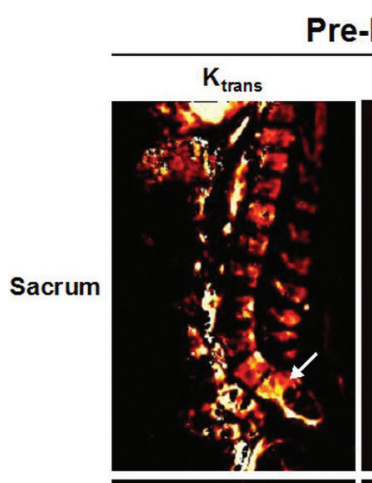

Pre-RT
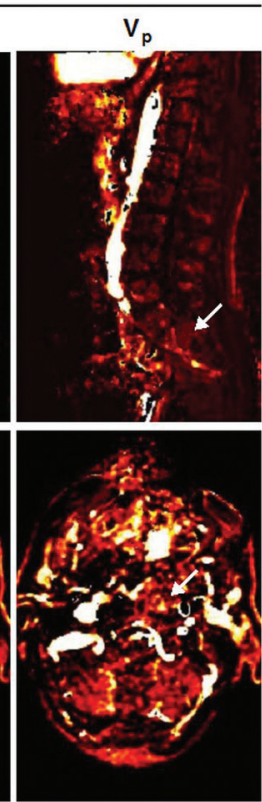

Post-RT
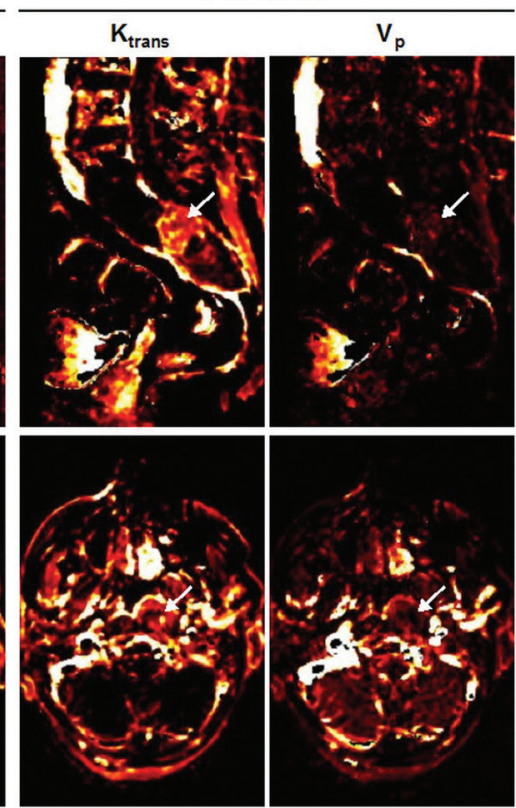

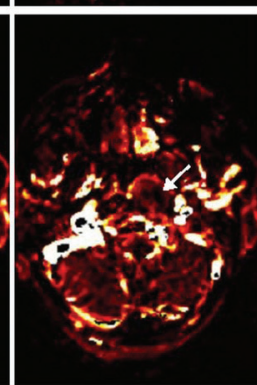

FIG 2. Perfusion maps of $K^{\text {trans }}$ and $V_{p}$ pre- and postradiation therapy. $K^{\text {trans }}$ and $V_{p}$ maps corresponding to the representative sacrum and clivus cases in Fig 1. Regions of enhancement are indicated by white arrows, both pre-RT and post-RT.

ment, 7 lesions exhibited moderate-to-high enhancement, while the remaining 4 lesions showed little-to-no enhancement. These findings were confirmed by corresponding $\mathrm{V}_{\mathrm{p}}$ and $K^{\text {trans }}$ maps, which also demonstrated similar enhancement patterns before RT (Fig 2).

Posttreatment, there was decreased enhancement across dynamic images (Fig 1A), as well as $\mathrm{V}_{\mathrm{p}}$ and $K^{\text {trans }}$ maps (Fig 2). Box-and-whisker representations illustrated significant differences in the perfusion parameters of chordoma lesions treated with RT (Fig 3). Of the 4 quantitative perfusion parameters, $V_{p}$ mean values showed the most significant difference $(P=.02)$ before $($ mean $=0.82)$ and after $($ mean $=0.42)$ treatment, followed by $\mathrm{V}_{\mathrm{p}}$ max (pre-RT mean $=3.56$; post-RT mean $=2.27$ ) and $K^{\text {trans }}$ mean values (pre-RT mean $=0.046$; post-RT mean $=$ 0.028 ), which also were significant with $P$ values of .04 and .03 , respectively (Fig 3 ). However, $K^{\text {trans }}$ max values showed no significant difference with RT (Fig 3).

\section{Dynamic Concentration-Time Curves}

Of the 11 time curves generated, 7 exhibited a persistent enhancement pattern pretreatment, while the remaining 4 curves exhibited a plateau pattern of enhancement (Fig 4B). Time curves following rapid contrast injection showed decreased signal intensity posttreatment in 9 of 11 cases (Fig 4B). Thereafter, individual curves were used to generate average time curves pre- and posttreatment (Fig 4A). Semiquantitative AUC analysis of the aggregate pre- and posttreatment curves showed a significant difference $(P=.03)$ in MR signal, decreasing from mean $=21.03$ pretreatment to mean $=14.52$ posttreatment $($ Fig $4 A)$.

\section{Perfusion and Long-Term Outcomes}

Several interesting clinical patterns emerged. First, all tumors imaged exhibited changes in at least 3 of the 5 quantitative $\left(V_{p} \max\right.$,
$\mathrm{V}_{\mathrm{p}}$ mean, $K^{\text {trans }} \max , K^{\text {trans }}$ mean, AUC) parameters. In contrast, only 4 irradiated tumors exhibited changes in quantitative size measurements on posttreatment conventional MR imaging. On average, the pretreatment DCE-MR imaging interval was 94.27 days before radiation and the posttreatment DCE-MR imaging interval was 153.81 days after radiation. In the 5 patients who later presented with local recurrence/tumor progression, concerning radiographic findings were detected on conventional MR imaging 346 days posttreatment on average. In comparison, significant changes in DCE-MR imaging were detectable 119.57 days posttreatment.

Second, 6 patients who received a shorter, more aggressive course of RT (24 Gy in 1 fraction) exhibited larger percentage changes in $\mathrm{V}_{\mathrm{p}}$ mean $($ mean $=-41.93), \mathrm{V}_{\mathrm{p}} \max ($ mean $=$ $-50.89), K^{\text {trans }}$ mean $($ mean $=-49.45)$, and $K^{\text {trans }} \max ($ mean $=-24.71)$ in comparison with patients who received longer, less aggressive courses of treatment $\left(\mathrm{V}_{\mathrm{p}}\right.$ mean $=-9.36, \mathrm{~V}_{\mathrm{p}}$ max mean $=-9.74, K^{\text {trans }}$ mean $=5.01$, and $K^{\text {trans }}$ max mean $=$ 36.66) (On-line Table 2).

Third, 7 patients with localized tumors had larger percentage decreases in $\mathrm{V}_{\mathrm{p}}$ mean (mean $=-52.07$ ) and $\mathrm{V}_{\mathrm{p}}$ maximum (mean $=-46.48)$ values in comparison with 4 patients with metastatic disease $\left(\mathrm{V}_{\mathrm{p}}\right.$ mean $=53.33, \mathrm{~V}_{\mathrm{p}}$ maximum mean $\left.=17.36\right)$, suggesting that localized tumors exhibited marked decreases in vascularity and thus were more responsive to RT. Likewise, 6 never-treated tumors had larger percentage decreases in $\mathrm{V}_{\mathrm{p}}$ mean $($ mean $=-55.02), \mathrm{V}_{\mathrm{p}} \max ($ mean $=-49.74)$, and $K^{\text {trans }}$ mean (mean $=-31.56)$ in comparison with 5 previously treated tumors, supporting previous findings that treatment-naïve tumors are more likely to respond to radiation therapy $\left(\mathrm{V}_{\mathrm{p}}\right.$ mean $=6.34$, $\mathrm{V}_{\mathrm{p}}$ max mean $=-11.12$, and $K^{\text {trans }}$ mean $\left.=-16.47\right)$ (On-line Table 2).

\section{DISCUSSION}

Conventional MR imaging of chordomas treated with RT typically exhibits little change. Our results suggest that DCE-MR imaging perfusion parameters obtained through quantitative $\left(\mathrm{V}_{\mathrm{p}}\right.$ and $K^{\text {trans }}$ ) and semiquantitative (AUC) analysis significantly changed following RT and may serve as a more sensitive indicator for monitoring tumor responsiveness to RT than conventional MR imaging.

\section{Major Findings}

Of the 3 parameters studied, $V_{\mathrm{p}}$ was the most significant measure of tumor response. Differences in $V_{p}$ mean and $V_{p}$ maximum values pre- and posttreatment were significant. On average, $V_{p}$ max values were approximately 1.6 times greater before treatment, which is concordant with previous work demonstrating the significantly higher vascularization of chordoma in comparison 


\section{$\mathrm{V}_{\mathrm{p}}$ mean}
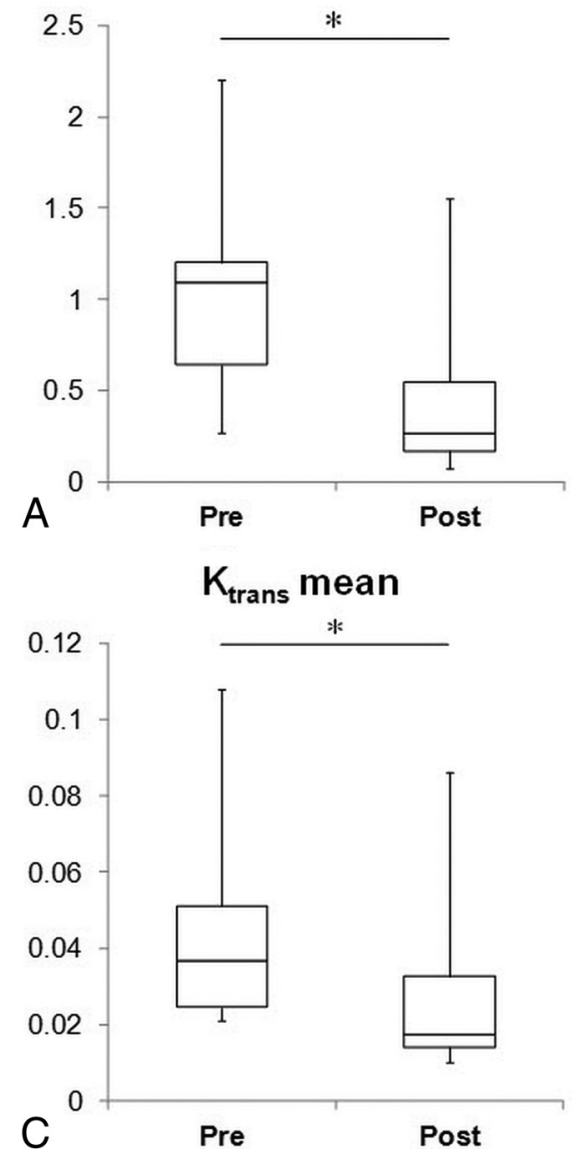

$\mathrm{V}_{\mathrm{p}} \max$
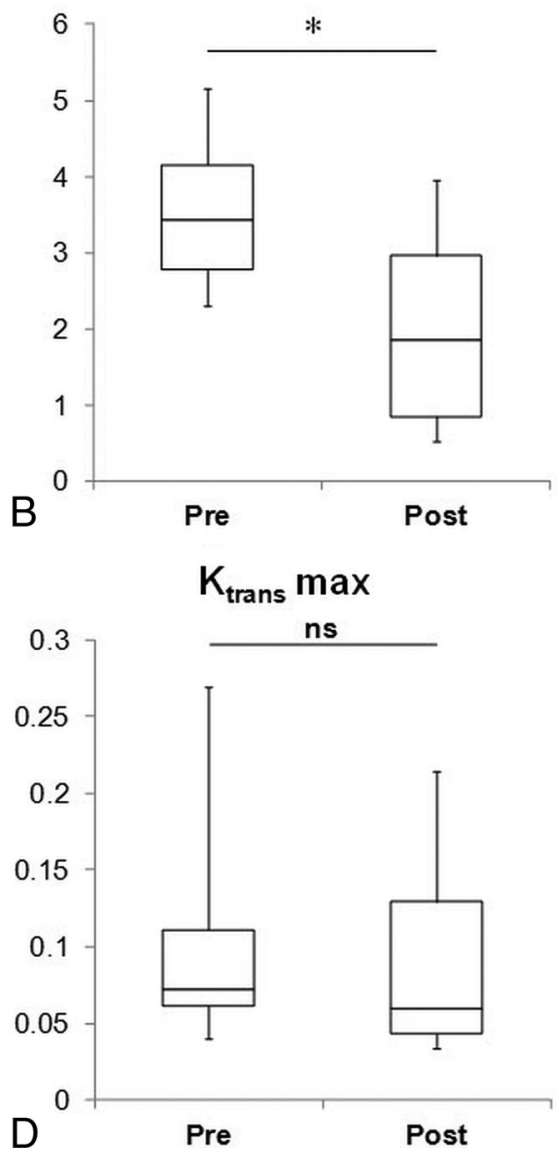

FIG 3. Tumor plasma volume and permeability decreased after radiation therapy. Box-and-whisker plots illustrating a five-number summary for DCE-MR imaging perfusion parameters: $A, V_{p}$ mean. $B, V_{p}$ max. $C, K^{\text {trans }}$ mean. $D, K^{\text {trans }} \max (n=12)$. Significant differences were observed pre-RT and post-RT in 3 of 4 parameters: $\mathrm{V}_{\mathrm{p}}$ mean (pre-RT mean $=0.82$, post-RT mean $=0.42$, asterisk indicates $P=.02$ ), $\mathrm{V}_{\mathrm{p}} \max$ (pre-RT mean $=3.56$, post-RT mean $=2.27$, asterisk indicates $P=.04$ ), and $K^{\text {trans }}$ mean (pre-RT mean $=0.046$, post-RT mean $=0.028$, asterisk indicates $P=.03$ ). Differences in $K^{\text {trans }} \max$ values pre-RT $($ mean $=0.11)$ and post-RT $($ mean $=0.092)$ were not significant $(P=.47)$.
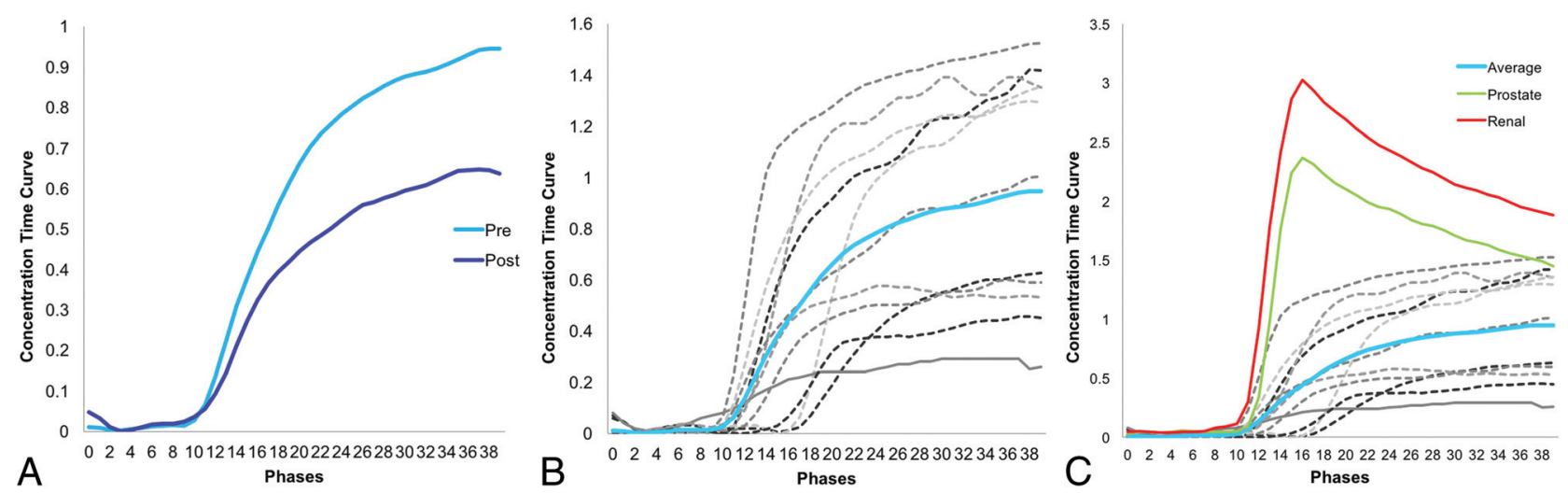

FIG 4. Chordoma is distinguished by its unique signature on DCE-MR signal intensity-time curves. A, Averaged dynamic MR signal intensity curves for patients with chordoma $(n=12)$ pre-RT (blue) and post-RT (purple). Significant differences in areas under the curve pre-RT and post-RT were observed $(P=.03)$. B, Individual dynamic MR signal intensity-time curves from 12 patients (gray) were used to generate an average curve (blue). C, Unlike hypervascular renal cell carcinomas (red) and hypovascular prostate carcinomas (green) spinal metastases, ${ }^{14}$ chordoma presents with a signal intensity-time curve characterized by slow contrast uptake and lack of peak enhancement, likely due to a slow washout phase. For all graphs, each phase $=1$ second. 
hancing" time-signal intensity curves before treatment (Fig $4 B,-C)$, a pattern of enhancement generally associated with more benign lesions. ${ }^{21}$ However, this pattern of contrast uptake is also consistent with low-grade tumors, such as chordomas. ${ }^{22}$ The remaining 3 of 11 patients with chordomas had pretreatment timesignal intensity curves that "plateaued" (Fig 4B, -C). Such curves are more suspicious for malignancy, but not definitive. ${ }^{22}$ While the pattern of enhancement remained the same for most patients, 2 patients exhibited changes in enhancement dynamics. It remains unclear whether enhancement dynamics offer a predictive value of treatment response.

Additionally, the persisting and plateauing kinetic patterns of enhancement on DCE-MR imaging are not unique to chordoma. Other osseous tumors of the spine, particularly chondrosarcoma and giant cell tumors, also exhibit similar patterns. ${ }^{23}$ Furthermore, factors such as the heterogeneous composition of a chordoma and the shape of the ROI may also account for the variation in kinetic patterns observed. ${ }^{9}$ Despite these uncertainties, semiquantitative AUC analysis of aggregate dynamic time-signal intensity curves demonstrated that RT was still associated with a significant decrease $(P=.03)$ in signal intensity posttreatment (Fig 4A), suggesting that AUC might be another parameter for characterizing chordoma response to RT.

Our findings are consistent with previous DCE studies that show that physiologic changes in tumor vascularity present earlier than corresponding morphologic changes. ${ }^{24,25}$ A representative case is patient 7. Although the initial posttreatment conventional MR imaging scan suggested that the tumor had responded to radiation therapy (ie, subjectively better), subsequent surveillance scans showed evidence of tumor progression, which was associated with clinical worsening as denoted by the corresponding radiation oncology progress notes. These findings provide evidence supporting DCE-MR perfusion imaging as an intriguing alternate to conventional MR imaging because decreases in detection time are crucial for rapid decision-making, particularly for patients with chordoma who may benefit from prompt treatment modifications. Our findings are also consistent with previous findings demonstrating that single-dose fractions of $>10$ Gy cause severe vascular damage, resulting in alterations of the tumor microenvironment and triggering tumor cell apoptosis. ${ }^{22}$ The cytotoxic effects of ionizing RT are well-established, with increasing doses leading to an increased risk of vascular pathologies. ${ }^{26}$ DCE-MR imaging can detect evidence of vascular damage via decreased $V_{p}$ and increased $K^{\text {trans }}$. Tumor staging and recurrence may have an influence on both prognostication and posttreatment DCE changes because both our study and previous studies ${ }^{27}$ have shown that tumor progression is associated with higher $\mathrm{V}_{\mathrm{p}}$ and $K^{\text {trans }}$ values.

There are several limitations to our study. First, larger sample sizes will permit ROC analysis and more estimation of the predictive value of perfusion parameters. Due to the exceedingly low incidence and prevalence of chordoma, our study was limited by the size of the available patient population. The sample size was further limited to patients with chordoma who received radiation therapy, pre- and posttreatment DCE perfusion scans, and no complicating operations. However, some clinical patterns did emerge that are worth investigating in future studies. Second, as a retrospective study, there was significant variability in the timing of the posttreatment DCE-MR images. Much of this variability could be attributed to numerous factors, including scheduling constraints of the patient and provider and technical errors resulting in suboptimal DCE-MR images that were excluded from the study. Notably, patients 7 and 11 had posttreatment DCE-MR images obtained after tumor progression was already detected on conventional MR imaging. Additionally, these patients presented with aggressive, widely metastatic disease that had already been previously irradiated. Collectively, these factors limit the predictive value of DCE-MR imaging in these 2 cases; thus, they were not included in the analysis of clinical outcomes.

Third, both the precontrast T1 value and arterial input function must be accurately measured to apply a pharmacokinetic model for quantitative analysis. In the design of DCE-MR imaging studies, competing demands for high spatial resolution, coverage, and signal-to-noise often resulted in inadequate temporal resolution for reliable measurement of the arterial input function. Fourth, the precise mechanism leading to the perfusion differences before and after RT has not been elucidated, and it is probable that the perfusion changes reflect a combination of radiationand therapy-induced changes, healing bone, and reconstitution of hematopoietic marrow. However, given that successfully treated chordomas could be differentiated by the perfusion changes by a statistically significant margin in our patient population, the results are meaningful regardless of the precise mechanism.

\section{CONCLUSIONS}

DCE-MR imaging presents a novel alternative to conventional MR imaging for tumor monitoring in patients with chordoma, providing insight into the physiologic and hemodynamic processes within a particular lesion. Quantitative and semiquantitative DCE parameters such as $\mathrm{V}_{\mathrm{p}}, K^{\text {trans }}$, and AUC are significantly decreased posttreatment; this change reflects the extent of vascular damage induced by radiation therapy. Collectively, these findings demonstrate the capacity of DCE-MR imaging to accurately reflect underlying tumor physiology in response to therapy.

Disclosures: Eric Lis_UNRELATED: Payment for Lectures Including Service on Speakers Bureaus: Medtronic. Yoshiya Yamada_UNRELATED: Board Membership: Chordoma Foundation Medical Advisory Board member, Comments: no financial remuneration; Payment for Lectures Including Service on Speakers Bureaus: BrainLab, Comments: Speakers Bureau. Andrei I. Holodny-UNRELATED: Grants/Grants Pending: National Institutes of Health, Comments: Multiple Principal Investigator on National Institutes of Health-National Institute of Biomedical Imaging and Bioengineering 1R01EB022720-01, Principal Investigator on a subaward for National Institutes of Health-National Institute of Biomedical Imaging and Bioengineering IR01EB022720-01, Principal Investigator on National Institutes of Health-National Cancer Institute R21 CA220144-01*; Other: President of fMRI Consultants, Comments: This is a purely educational company. *Money paid to the institution.

\section{REFERENCES}

1. Walcott BP, Nahed BV, Mohyeldin A, et al. Chordoma: current concepts, management, and future directions. Lancet Oncol 2012;13: e69-76 CrossRef Medline

2. Healey JH, Lane JM. Chordoma: a critical review of diagnosis and treatment. Orthop Clin North Am 1989;20:417-26 Medline

3. Stiller CA, Trama A, Serraino D, et al; RARECARE Working Group. Descriptive epidemiology of sarcomas in Europe: report from the RARECARE project. Eur J Cancer 2013;49:684-95 CrossRef Medline 4. McMaster ML, Goldstein AM, Bromley CM, et al. Chordoma: inci- 
dence and survival patterns in the United States, 1973-1995. Cancer Causes Control 2001;12:1-11 CrossRef Medline

5. Sung MS, Lee GK, Kang HS, et al. Sacrococcygeal chordoma: MR imaging in 30 patients. Skeletal Radiol 2005;34:87-94 CrossRef Medline

6. Smolders D, Wang X, Drevelengas A, et al. Value of MRI in the diagnosis of non-clival, non-sacral chordoma. Skeletal Radiol 2003;32: 343-50 CrossRef Medline

7. Rodallec MH, Feydy A, Larousserie F, et al. Diagnostic imaging of solitary tumors of the spine: what to do and say. Radiographics 2008; 28:1019-41 CrossRef Medline

8. Llauger J, Palmer J, Amores S, et al. Primary tumors of the sacrum: diagnostic imaging. AJR Am J Roentgenol 2000;174:417-24 CrossRef Medline

9. Chugh R, Tawbi H, Lucas DR, et al. Chordoma: the nonsarcoma primary bone tumor. Oncologist 2007;12:1344-50 CrossRef Medline

10. Stacchiotti S, Sommer J. Building a global consensus approach to chordoma: a position paper from the medical and patient community. Lancet Oncol 2015;16:e71-83 CrossRef Medline

11. Fuchs B, Dickey ID, Yaszemski MJ, et al. Operative management of sacral chordoma. J Bone Joint Surg Am 2005;87:2211-16 Medline

12. Parker G, Buckley D. Tracer kinetic modeling for T1-weighted DCEMRI. In: Jackson A, Buckley D, Parker G. Dynamic Contrast-Enhanced Magnetic Resonance Imaging in Oncology. Berlin: Springer; 2005:81-91

13. Chu S, Karimi S, Peck KK, et al. Measurement of blood perfusion in spinal metastases with dynamic contrast-enhanced magnetic resonance imaging: evaluation of tumor response to radiation therapy. Spine 2013;38:E1418-24 CrossRef Medline

14. Saha A, Peck KK, Lis E, et al. Magnetic resonance perfusion characteristics of hypervascular renal and hypovascular prostate spinal metastases: clinical utilities and implications. Spine 2014;39: E1433-40 CrossRef Medline

15. Lang N, Su MY, Xing X, et al. Morphological and dynamic contrast enhanced MR imaging features for the differentiation of chordoma and giant cell tumors in the axial skeleton. J Magn Reson Imaging 2017;45:1068-75 CrossRef Medline

16. Tofts PS, Brix G, Buckley DL, et al. Estimating kinetic parameters from dynamic contrast-enhanced T(1)-weighted MRI of a diffus- able tracer: standardized quantities and symbols. J Magn Reson Imaging 1999;10:223-32 Medline

17. Chen KW, Yang HL, Lu J, et al. Expression of vascular endothelial growth factor and matrix metalloproteinase-9 in sacral chordoma. J Neurooncol 2011;101:357-63 CrossRef Medline

18. Kawashima A, Okada Y, Nakanishi I, et al. Immunolocalization of matrix metalloproteinases and tissue inhibitors of metalloproteinases in human chondrosarcomas. Gen Diagn Pathol 1997;142: 129-37 Medline

19. Kumta SM, Huang L, Cheng YY, et al. Expression of VEGF and MMP-9 in giant cell tumor of bone and other osteolytic lesions. Life Sci 2003;73:1427-36 CrossRef Medline

20. Kaya M, Wada T, Kawaguchi S, et al. Increased pre-therapeutic serum vascular endothelial growth factor in patients with early clinical relapse of osteosarcoma. Br J Cancer 2002;86:864-69 CrossRef Medline

21. Johnson LM, Turkbey B, Figg WD, et al. Multiparametric MRI in prostate cancer management. Nature Rev Clin Oncol 2014;11: 346-53 CrossRef

22. Park HJ, Griffin RJ, Hui S, et al. Radiation-induced vascular damage in tumors: implications of vascular damage in ablative hypofractionated radiotherapy (SBRT and SRS). Radiat Res 2012;177:311-27 CrossRef Medline

23. De Coninck T, Jans L, Sys G, et al. Dynamic contrast-enhanced MR imaging for differentiation between enchondroma and chondrosarcoma. Eur Radiol 2013;23:3140-52 CrossRef Medline

24. Røe K, Kakar M, Seierstad T, et al. Early prediction of response to radiotherapy and androgen-deprivation therapy in prostate cancer by repeated functional MRI: a preclinical study. Radiat Oncol 2011; 6:65 CrossRef Medline

25. Cao Y. The promise of dynamic contrast-enhanced imaging in radiation therapy. Semin Radiat Oncol 2011;21:147-56 CrossRef Medline

26. Grabham $P$, Sharma P. The effects of radiation on angiogenesis. Vasc Cell 2013;5:1 CrossRef Medline

27. Thomas AA, Arevalo-Perez J, Kaley T, et al. Dynamic contrast enhanced T1 MRI perfusion differentiates pseudoprogression from recurrent glioblastoma. J Neurooncol 2015;125:183-90 CrossRef Medline 